\title{
Adaptive Filtering for Indoor Localization using ZIGBEE RSSI and LQI Measurement
}

\author{
Sharly Joana Halder ${ }^{1}$, Joon-Goo Park ${ }^{2}$ and Wooju Kim ${ }^{1}$ \\ ${ }^{1}$ Yonsei University, Seoul \\ ${ }^{2}$ Kyungpook National University, Daegu \\ Republic of Korea
}

\section{Introduction}

The term "filter" is often used to describe a device in the form of a piece of physical hardware or computer software that is applied to a set of noisy data in order to extract information about a prescribed quantity of interest [25], [26]. Filter has been designed to take noisy data as input to reduce the effects of noise as much as possible.

A Wireless Sensor Network (WSN) is a network that consists of numerous small devices that are in fact tiny computers. These so-called nodes are composed of a power supply, a processor, different kinds of memory and a radio transceiver for communication. WSNs are generally used to observe or sense the environment in a non-intrusive way. In order to perform this task, nodes are often extended with sensors, like infrared, ultrasonic or temperature sensors, hence the names sensor nodes and sensor networks. The domain of WSNs is still very young. During the last few years, new developments in the area of communication, computing and sensing have enabled and stimulated the miniaturization and optimization of computer hardware. These evolutions have led to the emergence of WSNs. Despite the increasing capabilities of hardware in general, sensor nodes are still very restricted devices. They have a limited amount of processing power, memory capacity and most importantly energy. This makes WSNs a challenging research topic.

Despite current restrictions, several applications for WSNs have already been designed. WSNs are currently found in very different domains [3]. The large literature can be classified by relying on several criteria. One of these is the physical means used for localization, e.g., through the RF attenuation in the Electro-Magnetic (EM) waves [4], [11], [13] (Received Signal Strength Indicator - RSSI - based techniques) or the time required to cover the distance between transmitter and receiver (Ultra Wide Band); if using ultrasonic pulses, one could also use the time of arrival or time-difference of arrival of the waves [10]. This can even be extended to Audible-frequency sounds [9]. Another classification is based on the ranging feature, where distinguish between Range-free and Range-based localization techniques [11]. Moreover, it can be classified according to the Single-hop [11] and Multihop [14] localization scheme. Finally, it can differentiate between centralized [14] and distributed [9] localization systems.

A common consensus among localization researchers is that indoor localization requires room-level accuracy. Indoor localization uses many different sensors such as infrared, RFID, Ultrasound, Ultra-Wide Band, Bluetooth, and WLAN. Different sensors provide different 
range of accuracy from centimeters to room-level. It seems like the accuracy is smaller than a room. But in practice, when we directly transform $(x, y)$, it coordinates to room-level information and causes mistakes. The reason being is, wireless signal is easy to suffer disturbance that makes localization unusual. This causes jumping in a split second or over a short span. Such situation may effect the location estimation from one room to another.

Ubiquitous indoor environments often contain substantial amounts of metal and other such reflective materials that affect the propagation of radio frequency signals in non-trivial ways, causing severe multipath effects, dead-spots, noise and interference. The main focus of this scheme is to represents a cheap and enhanced ranging technique to measure the radio strength by using two useful radio hardware link quality metrics named Link Quality Indicator (LQI) and Received Signal Strength Indicator (RSSI). In this scheme the mobile device itself calculate the position. Moreover, the device calculates its own position based on its own measurements. The proposed protocol tries to improve the existing algorithms [4], [27] using RSSI and LQI values. The indoor localization systems presented in this report are based on the RSSI as a strength indicator and LQI as a quality indicator of received packets. It can also be used to estimate a distance from a node to reference points. This system uses the LQI and RSSI in a different way and therefore it could lead to better and more predictable results than the other existing system. Several experiments were conducted to investigate the performance of the proposed scheme. At first, this system performs with respect to the signal analysis to understand the characteristic of the LQI and RSSI values on three types of environments to decide how the environment effects on RSSI and LQI strength. The effect of distance on received signal strength can be measured by RSSI and LQI provided by the radio. Secondly, this scheme performs with respect to the signal analysis is to filter the original signals in order to remove the noise. Besides, the noise could be estimated by using adaptive filtering algorithms. Sudden peaks and gaps in the signal strength are removed and the whole signal is smoothed, which eases the analysis process. We used two different types of new filtering to smooth the real RSSI, 'LQI' filtering and 'BOTH' filtering, and compared the results. And we found that ' $\mathrm{BOTH}$ ' filter smooth more the raw RSSI value than existing 'Fusion' filtering [27].

In our research we used an adaptive filter as it performs well to track an object under such changing conditions in the RF signal environment. In this chapter, the proposed protocol will try to improve the existing algorithms using RSSI and LQI values. The localization systems presented in this report are based on RSSI as a strength indicator and LQI as a quality indicator of a received packets, it can also be used to estimate a distance from a node to reference points.

The remainder of this Chapter consists of six sub chapters. Chapter 2 describes some properties of ZigBee, RSSI and LQI. Chapter 3 reveals the previous works based on indoor location and WSN. Chapter 4 provides the proposed model of "Adaptive Filtering for Indoor Localization using ZIGBEE RSSI and LQI Measurement" and its probability of returning the correct location. Chapter 5 describes the analytical results obtained from the model of location system. And Chapter 6 concludes the chapter with conclusions.

\section{ZigBee, RSSI and LQI}

\subsection{ZigBee}

There are several standards that address mid to high data rates for voice, PC LANs, video, etc. and until recently there has not been a wireless network standard that meets the unique 
needs of devices such as sensors and control devices. Sensors and control devices which are mostly used in industries and homes distinguish them with low data rates and in needs of very low energy consumption. A standards-based wireless technology needed having the performance characteristics that closely meet the requirements for reliability, security, low power and low cost.

Table 1 presented the IEEE 802.15 Task Group 4 is chartered to investigate a low data rate solution with multi-month to multi-year battery life and very low complexity. It is intended to operate in an unlicensed, international frequency band.

Since low total system cost is a main issue in industrial and home wireless applications, a highly integrated single-chip approach is the preferred solution of semiconductor manufacturers developing IEEE 802.15.4 compliant transceivers. The IEEE standard at the PHY is the significant factor in determining the RF architecture and topology of ZigBee enabled transceivers. For these optimized short-range wireless solutions, the other key element above the Physical and MAC Layer is the Network/Security Layers for sensor and control integration. The ZigBee group was organized to define and set the typical solutions for these layers for star, mesh, and cluster tree topologies.

\begin{tabular}{|c|c||c|c|}
\hline Feature(s) & IEEE 802.11b & Bluetooth & ZigBee \\
\hline Power Profile & Hours & Days & Years \\
\hline Complexity & Very Complex & Complex & Simple \\
\hline Nodes/Master & 32 & 7 & 64000 \\
\hline \hline Latency & Enumeration upto 3 sec & Enumeration upto $10 \mathrm{sec}$ & Enumeration $30 \mathrm{~ms}$ \\
\hline \hline Range & $100 \mathrm{~m}$ & $10 \mathrm{~m}$ & $70 \mathrm{~m} 300 \mathrm{~m}$ \\
\hline \hline Extendability & Roaming possible & No & Yes \\
\hline Data Rate & $11 \mathrm{Mbps}$ & $1 \mathrm{Mbps}$ & 250 Kbps \\
\hline Security & $\begin{array}{c}\text { Authentication Service } \\
\text { Set ID (SSID) }\end{array}$ & 64 bit, 128 bit & $\begin{array}{c}\text { Application Layer } \\
\text { user defined }\end{array}$ \\
\hline
\end{tabular}

Table 1. Comparison of key features of complementary wireless technologies [4]

\section{ZigBee Applications:}

ZigBee is the wireless technology that:

- Enables broad-based deployment of wireless networks with low cost, low power solutions [5].

- Provides the ability to run for years on inexpensive primary batteries for a typical monitoring application [5].

- Addresses the unique needs of remote monitoring \& control, and sensory network applications [5].

Figure 1 shows the ZigBee application areas. However, ZigBee technology is well suited to a wide range of building automation, industrial, medical and residential control \& monitoring applications. Essentially, applications that require interoperability and/or the RF performance characteristics of the IEEE 802.15.4 standard would benefit from a ZigBee solution. 


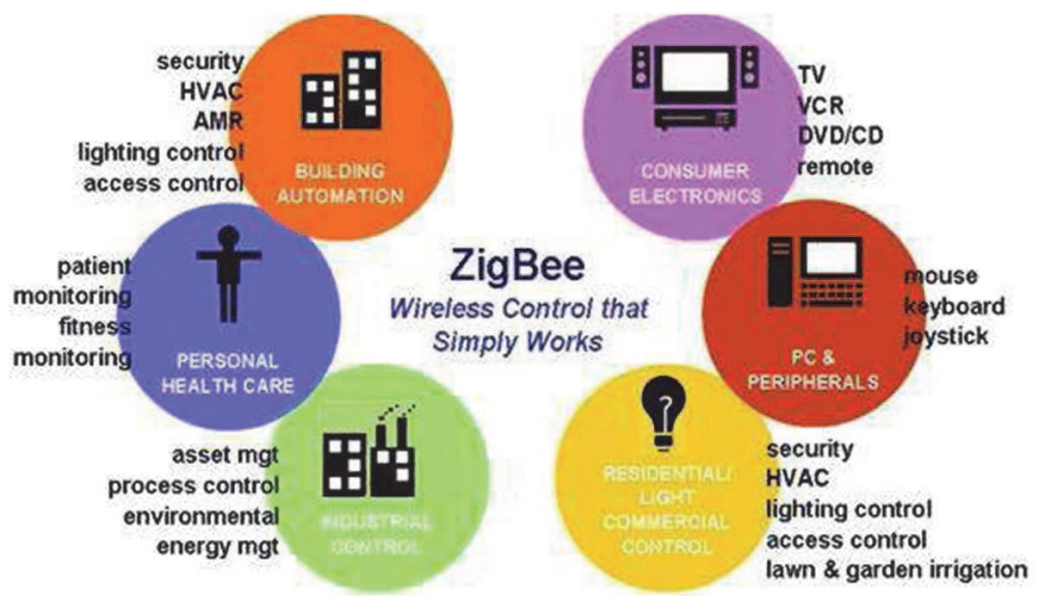

Fig. 1. ZigBee applications [5].

\subsection{Received signal strength indicator (RSSI)}

Majority of the existing methods leverage the existence of IEEE 802.11 base stations with powerful radio transmit powers of approximately $100 \mathrm{~mW}$ per base station. Such radios are in a different class from the low power IEEE 802.15.4 compliant radios that typically transmit at low power levels ranging from $52 \mathrm{~mW}$ to $29 \mathrm{~mW}$. The wide availability of larger number of IEEE 802.15.4 radios has revived the interest for signal strength based localization in sensor network. Despite of rapidly increasing popularity of IEEE 802.15.4 radios and signal strength localization, there is a lack of detailed characterization of the fundamental factors contributing to large signal strength variation. The analysis of RSSI values is needed to understand the underlying features of location dependent RSSI patterns and location fingerprints. An understanding of the properties of the RSSI values for location can assist in improving the design of positioning algorithms and in deployment of indoor positioning systems. The characteristics of RSS, received signal strength will decrease with increased distance as the equation below shows:

$$
\text { RSSI }=-\left(10 \log _{10} \mathrm{~d}+\mathrm{A}\right)
$$

Where,

$\mathrm{n}=$ signal propagation constant, also named propagation exponent.

$\mathrm{d}=$ distance from sender.

$\mathrm{A}=$ received signal strength at a distance of one meter.

Lots of localization algorithms require a distance to estimate the position of unknown devices. One possibility to acquire a distance is measuring the received signal strength of the incoming radio signal.

The idea behind RSS is that the configured transmission power at the transmitting device $(P T X)$ directly affects the receiving power at the receiving device (PRX). According to Friis' free space transmission equation, the detected signal strength decreases quadratically with the distance to the sender (Figure 2.a).

$$
P R X=P T X * G T X * G R X(\lambda / 4 \Pi d)^{2}
$$




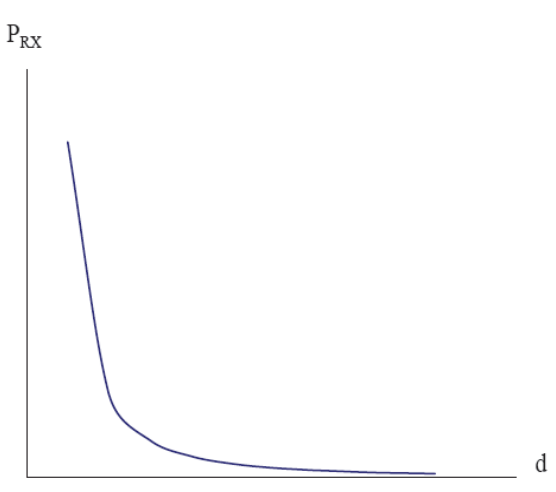

(a)

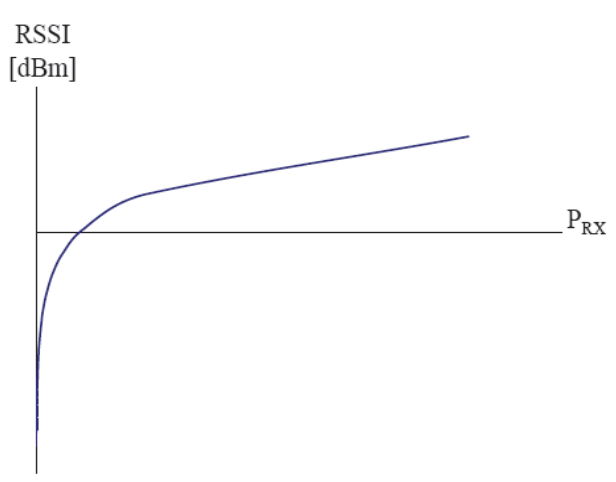

(b)

Fig. 2. (a) Received power PRX versus distance to the transmitter. (b) RSSI as quality identifier of the received signal power PRX.

Where,

PTX $=$ Transmission power of sender

$\mathrm{PRX}=$ Remaining power of wave at receiver

GTX $=$ Gain of transmitter

GRX $=$ Gain of receiver

$\lambda=$ Wave length

$\mathrm{d}=$ Distance between sender and receiver

In embedded devices, the received signal strength is converted to a received signal strength indicator (RSSI) which is defined as ratio of the received power to the reference power (PRef). Typically, the reference power represents an absolute value of Pref $=1 \mathrm{~mW}$.

$$
\mathrm{RSSI}=10 * \log \mathrm{PRX} / \mathrm{PRF} \quad[\mathrm{RSSI}]=\mathrm{dBm}
$$

An increasing received power results a rising RSSI. Figure 2.b illustrates the relation between RSSI and the received signal power. Plotting RSSI versus distance $d$ results in a graph, which is in principle axis symmetric to the abscissa. Thus, distance $d$ is indirect proportional to RSSI. In practical scenarios, the ideal distribution of $P R X$ is not applicable, because the propagation of the radio signal is interfered with a lot of influencing effects.

\subsection{Link quality indicator (LQI)}

For communications IEEE 802.15.4 radios provide applications with information about the incoming signal [17]. The effect of distance on received signal strength (RSS) can be measured by the packet success rate, RSSI and LQI provided by the radio. LQI is a metric introduced in IEEE 802.15.4 that measures the error in the incoming modulation of successfully received packets (packets that pass the CRC criterion). The LQI metric characterizes the strength and quality of a received packet. It is introduced in the 802.15.4 standard [1] and is provided by CC2430 [17]. LQI measures each successfully received packet and the resulting integer ranges from 0x00 to 0xff (0-255), indicating the lowest and highest quality signals detectable by the receiver (between $-100 \mathrm{dBm}$ and $0 \mathrm{dBm}$ ). The correlation value of LQI range from 50 to 110 where 50 indicates the minimum value and 
110 represents the maximum. The 50 is typically the lowest quality frames detectable by CC2430. Software must convert the correlation value to the range $0-255$, e.g. by calculating:

$$
\mathrm{LQI}=(\mathrm{CORR}-\mathrm{a}) \cdot \mathrm{b}
$$

Where,

$\mathrm{CORR}=$ correlation value, $\mathrm{a}$ and $\mathrm{b}$ are found empirically

The CORR (correlation value) is the raw LQI value which can be obtained from the last byte of the message. The raw value can get from CC2430 (CORR) is between 40 and 110.

Limited to the range $0-255$, where $a$ and $b$ are found empirically based on PER measurements as a function of the correlation value. A combination of RSSI and correlation values may also be used to generate the LQI value. LQI values are uniformly distributed between these two limits. Different form RSSI, LQI measures the qualities of links while RSSI measures the strengths of links. LQI is a measure of the error in the signal, not the strength of the signal. A "weak" signal may still be a very crisp signal with no errors and thus a potentially good routing neighbor. If there is no interference from other $2.4 \mathrm{GHz}$ devices, then LQI will generally be good over distance. Note that, scaling the link quality to a LQI, compliant with IEEE 802.15.4, must be done by software. This can be done on the basis of the RSSI value, the correlation value or a combination of those two. Signal strength and link quality values are not necessarily linked. But if the LQI is low, it is more likely that the RSSI will be low as well. Nevertheless, they also depend on the emitting power. A research group had the following results:

\begin{tabular}{|c|c|c|}
\hline & Low RF & High RF \\
\hline LQI & 105 & 108 \\
\hline RSSI & $-75 \mathrm{dBm}$ & $-25 \mathrm{dBm}$ \\
\hline
\end{tabular}

Even though they do not describe how far from each other the sender and the receiver are located, it illustrates perfectly that both low and high power emissions guarantee a good link quality. The low RF emissions could be more sensitive to external disturbances. LQI exhibits a very good correlation with packet loss, and is therefore a good link quality indicator. However, one of the contributions of the present work is to show that RSSI is a reasonable metric if it is processed correctly, and if interference can be distinguished from noise. Given that LQI is a superior metric, it should not be forgotten that it is only made available by 802.15 .4 -compliant devices. It therefore makes sense to make the most out of RSSI.

\section{Related works}

This chapter introduces the area of ubiquitous computing and the underlying sensing technologies such as ultrasonic, infrared, Global Positioning Systems, and radio frequency identification. At first, an brief overview of each of the systems is given and then the similarities and differences to the approach are discussed.

\subsection{Terminology and principles}

There are numbers of existing location systems which utilize a variety of sensing technologies and system architectures. These systems have varying characteristics, such as accuracy, scalability, range, power consumption and cost. This section describes some of the 
sensor terminology and principles used with reference to location systems. There are a number of different sensor technologies have been used in location systems.

\subsubsection{Light}

Light is a widely-used medium in location systems, varying from the use of simple infrared LEDs and sensors to tag-less vision based tracking. Infrared has been popularly used for containment-based location systems [20]. Infrared location systems can suffer in strong sunlight and under fluorescent tube lighting as both of these are sources of infrared light. Video cameras can be used both to recognize objects within the environment, allowing the device to calculate its position, or as an infrastructure to track mobile objects which may or may not be augmented. The processing power required to track objects, especially if they are untagged, using image-based methods can be large compared to other methods.

\subsubsection{Radio-based localization}

Localization in sensor networks can be achieved using knowledge about the radio signal behavior and the reception characteristics between two different sensor nodes. The quality of a radio signal, i.e. its strength at reception time, is expressed by RSSI: the higher the RSSIvalue, the better the signal reception. The main advantage of using radio-based localization techniques is that no additional hardware for the sensor nodes is required. The main disadvantage of the technique is that the measured signal strengths are generally unstable and variable over time, which leads to localization errors. In this section, two common localization techniques using radio signal strength information are presented. Afterwards, the proximity idea is discussed, a technique that takes into account the range of radio communication rather than its quality. Finally, a technique for analyzing the RSSI behavior over time is presented. The technique cannot be used for localization itself, but it can provide useful mobility information about the node to be located. Following are three types of radio-based localization systems:

\subsubsection{Converting signal strength to distance}

In theory, there exists an exponential relation between the strength of a signal sent out by a radio and the distance the signal has traveled. In reality, this correlation has proven to be less perfect, but it still exists. Reference nodes broadcast a message to inform their position at regular intervals. Unknown nodes receive the broadcast message from reference nodes and measure the strength of the received signal [4], [27], [28]. Localization errors for this method range from two to three meters at average, with indoor errors being larger than outdoor ones. The main reason for the large number of errors is that the effective radiosignal propagation properties differ from the perfect theoretical relation that is assumed in the algorithm. Reflections, fading and multipath effects largely influence the effective signal propagation. The distance estimates, which are based on the theoretical relation, are thus inaccurate and lead to high errors in the calculated locations.

\subsubsection{Fingerprinting signal strength}

The second method that uses RSSI for localization is called Fingerprinting. This technique is based on the specific behavior of radio signals in a given environment, including reflections, fading and so on, rather than on the theoretical strength-distance relation. The fingerprinting technique [11], [12], [15] is an anchor-based technique that consists of two separate phases. During the first phase, called the Offline Phase, a fingerprint database of 
the environment is constructed. During the next phase, called the Online Phase, real-time localization is performed. The greatest disadvantage of the fingerprints method is that an offline phase is required for the system to work. The offline phase is very time consuming. Moreover, the fingerprinting database that is created during the offline phase is location dependent. If one wants to use the same system in another environment or if radical changes to the current environment are made, the offline phase has to be repeated.

\subsubsection{Proximity-based localization}

Proximity-based localization systems are an anchor-based solution to the localization problem. These systems derive their location data from connectivity information of the network [4], [7], [8], [11], [12], [23]. Knowledge about whether two devices, i.e. an unknown node and an anchor, in the network are within communication range is transformed into an assumption about their mutual distance and location.

\subsubsection{Ultrasound}

The propagation speed of ultrasound waves in air is slow compared to that of RF. Sound waves are generally reflected by objects in the environment, which also makes position by containment possible. Utilizing the differential time-of-flight between RF and ultrasound pulses allows position to be estimated to within a few centimeters of the ground truth. The attenuation of sound in air limits range to several meters. Sound waves generally take about $20 \mathrm{~ms}$ to die out; this therefore limits the update rate ultrasound location systems can obtain. The prevalent frequency used in ultrasound ranging is ultrasound. A lot of ultrasound location systems have been developed using narrow band $40 \mathrm{kHz}$ transducers [10], [21]. Following are three types of ultrasound based localization systems:

\subsubsection{The bat ultrasonic location system}

Bat system provides fine-grain 3D location and orientation information which its predecessor, the Active Badge System, did not. Position is calculated using trilateration. The Bat emitter will transmit a short ultrasound pulse and receivers placed at $1.5 \mathrm{~m}$ apart at known locations on the ceiling will pick up the signals [16].

\subsubsection{Cricket}

Cricket [10] is an indoor location system developed at MIT and utilizes RF and ultrasound using static transmitters and mobile receivers. The first iteration of the system is containment-based allowing for areas of arbitrary size to be created via careful placement of transmitters in the environment. A later iteration, called Cricket Compass [22], set out to allow orientation as well as position to be determined.

\subsubsection{Dolphin}

The Dolphin system [21], developed at the University of Tokyo, utilizes both RF and ultrasound to create a peer-to-peer system, providing co-ordinate based positioning. The aim is to develop a system which is easy to configure and provides a high degree of accuracy in three dimensions.

\subsubsection{Adhoc positioning system (APS) using AoA}

Niculescu and Nath [6] aim to create an algorithm and simulate a system where nodes have highly directional detection capabilities and there exist a small number of seeded nodes. Different algorithms were simulated in this chapter to gain some insight into how systems 
with different properties would behave. The data suggests that higher node densities increase the probability of node connectivity sufficiently to calculate location and orientation. Smaller angles lower the error. However this reduces the percentage of nodes for which locations are estimated.

\subsubsection{Global positioning system (GPS)}

The GPS system consists of twenty seven satellites that orbit the Earth [14], [24]. GPS use the distance and angle measurements to the reference points are used to compute the position of the object by triangulation. GPS uses the time of flight of RF signals to estimate the distance between GPS satellites and receiver [24]. In indoor environments, GPS satellites signals get attenuated and reflected by various metallic objects [24]. Indoor GPS performance has fundamental limitations that result in much larger position estimation errors compared to outdoors.

RSSI can provide us with the cheapest localization system possible, while the form factor of the sensor nodes is not increased. The technique is applicable to indoor environment and the errors achieved with a RSSI-based system seem to be promising compared to the more expensive systems. In this chapter, we decided to design and implement a RSSI-based system to solve the localization problem listed above. The main reason is that it can be developed with small modifications to the existing systems.

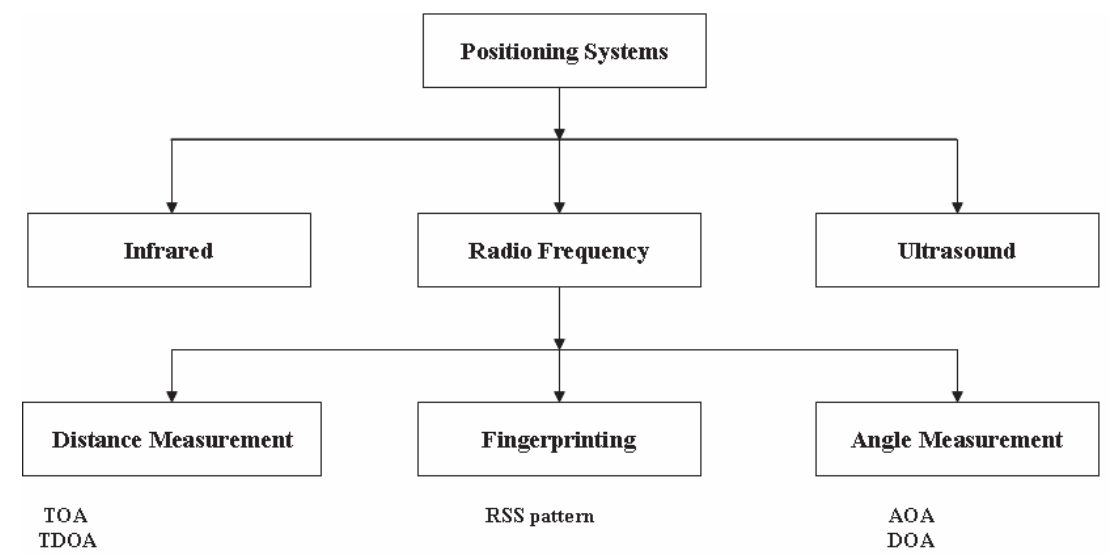

Fig. 3. Taxonomy of positioning system.

\subsubsection{Comparison}

The following table provides a comparison of the surveyed sensing systems from the point of accuracy and precision, scale, cost and limitations:

From Table 2, we concluded that RSSI can provide us with the cheapest localization system possible, while the form factor of the sensor nodes is not increased. The technique is applicable to indoor environment and the errors achieved with a RSSI-based system seem to be promising compared to the more expensive systems. In this chapter, we decided to design and implement a RSSI-based system to solve the localization problem listed here. The main reason is that it can be developed with small modifications to the existing systems. However, the aspects of accuracy and coverage area are still to be investigated in Chapter 4 and 5 . 


\begin{tabular}{|c|c|c|c|c|}
\hline Applicable indoor & GPS & Infrared & Ultrasound & RSSI \\
\hline $\begin{array}{c}\text { Need for extra } \\
\text { hardware }\end{array}$ & Yes & Yes & Yes & Yes \\
\hline $\begin{array}{c}\text { Cost of extra } \\
\text { hardware }\end{array}$ & High & Low & High & No \\
\hline $\begin{array}{c}\text { Size of extra } \\
\text { hardware }\end{array}$ & Average & Average & Large & N.A \\
\hline $\begin{array}{c}\text { Average expected } \\
\text { error }\end{array}$ & \pm 10 meters & \pm 5 meters & \pm 10 meters & $1 \sim 3$ meters \\
\hline
\end{tabular}

Table 2. Comparison of different location sensing technologies [13]

\section{Proposed scheme}

This chapter will focus on how this effective protocol has been implemented, and implementation issues are considered. In our experiments, to measure the radio strength, two useful radio hardware link quality metrics were used: (i) LQI and (ii) RSSI. Specifically, RSSI is the estimate of the signal power and is calculated over 8 symbol periods, while LQI can be viewed as chip error rate and is calculated over 8 symbols following the start frame delimiter (SFD). The specific point in a system where position estimates are calculated is an important design parameter. In this scheme the mobile device itself calculate the position. The device calculates its own position based on its own measurements.

\subsection{Selected location system architecture}

This scheme decides to use a private and scalable system. It features an active base station that transmits both RSSI and LQI signals. The mobile devices receive the signals, but they do not transmit anything themselves. The base station transmits the RSSI and LQI signals at the same moment in time. A mobile device measures signal, and is able to calculate the distance to the transmitter. By this scheme the location privacy of the user, who carries the mobile device, can be easily guaranteed because the mobile device does not send out any signals that might disclose its presence or its location. A further advantage of this architecture is scalability to many mobile devices. Because the mobile devices do not transmit any signals, there can be an unlimited number of mobile devices in principle. Due to its privacy and scalability features, this architecture might be particularly suitable for large-scale professional location systems or systems in public spaces. Each mobile device calculates its own position, based on the received signals. This scheme has divided into two subsystems.

As we know, for environmental changes the log model also change, so the proposed system uses a scaling factor for adjusting the log model with the measured data. This system includes a scaling factor s with the basic RSSI log model equation.

$$
\text { RSSI }=-10 n \log _{10}(\mathrm{sd}+1)+\mathrm{A}
$$

Where,

$\mathrm{s}=$ scaling factor

For our experiment, we use a filtering process for smoothing the RSSI values. We proposed a LQI filtering and BOTH filtering of RSSI and LQI values, for smoothing the measured 
RSSI. From our experiment, we determine the filtering factor $a$ for filtering and we used the following equation for smoothing the measured RSSI.

$$
\text { smooth_RSSI } \mathrm{t}_{\mathrm{(}(\mathrm{BOTH})}=\mathrm{a} * \operatorname{RSSI}_{\mathrm{t}}+(1-\mathrm{a}) * \mathrm{RSSI}_{\mathrm{t}-1}
$$

\section{Experimental result}

Adaptive filter contains a set of adjustable parameters. In design problem the requirement is to find the optimum set of filter parameters from knowledge of relevant signal characteristics according to some criterion.

This mathematical system combines the general principles of a proximity-based localization system with the analysis of the radio signal strength behavior over distance. This system uses the link quality indicator and radio signal strength indicator in a different way and therefore it could lead to better and more predictable results than the other existing system. Several experiments had conducted to investigate the performance of the proposed scheme.

The first step of this system performs with respect to the signal analysis to understand the characteristic of LQI and RSSI values on three types of environments. The effect of distance on received signal strength can be measured by RSSI and LQI provided by the radio. Equation 1 describes the basic model formula for RSSI where RSS decrease with increased distance. As for environmental change the log model also changed, this scheme decided to use a scaling factor $\mathrm{s}$ in the basic log model equation to adjust the log model with measured RSSI values. So, to find the accurate log model for specific environment we use a scaling factor $\mathrm{s}$ in equation 5 . The experiment is conducted on three types of following environment to decide how the environment effects on RSSI and LQI strength. The first experiment is conducted in close space indoor environment.

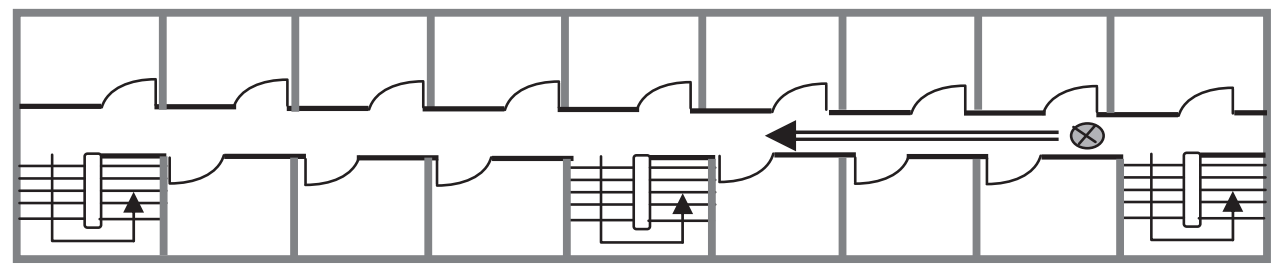

Fig. 4. Close space indoor environment (path 1).

The second experiment is deployed in half open space indoor environment, where few meters of the corridor was open.

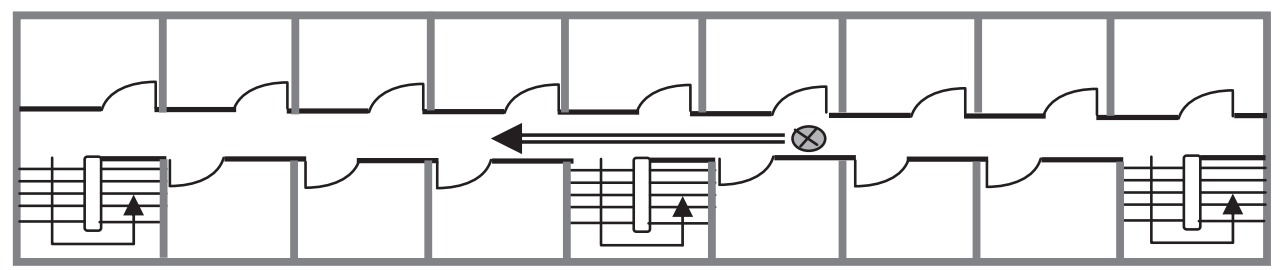

Fig. 5. Half open space indoor environment (path 2). 
The third experiment is conducted on the open space indoor environment to decide the variation of the RF and LQI from other two experiments.

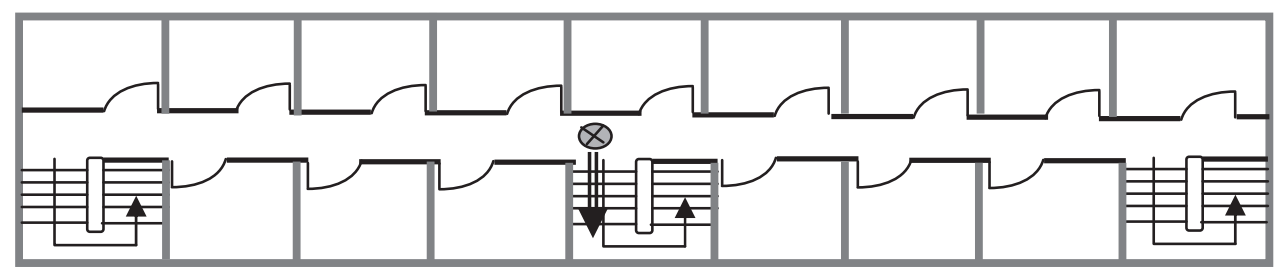

Fig. 6. Open space indoor environment (path 3).

To determine the accurate distance, the following distance equation has been used:

$$
\text { Distance }=\frac{\frac{R S S I-A}{10-10 n}-1}{s}
$$

And for measuring the signal attenuation factor the following equation has used:

$$
N=\frac{R S S I-A}{-10 \log 10(s d+1)}
$$

The following figures represent the measured RSSI and LQI values. These figures are also representing how the log model curve adjusted with the measured RSSI values in close space indoor environments.
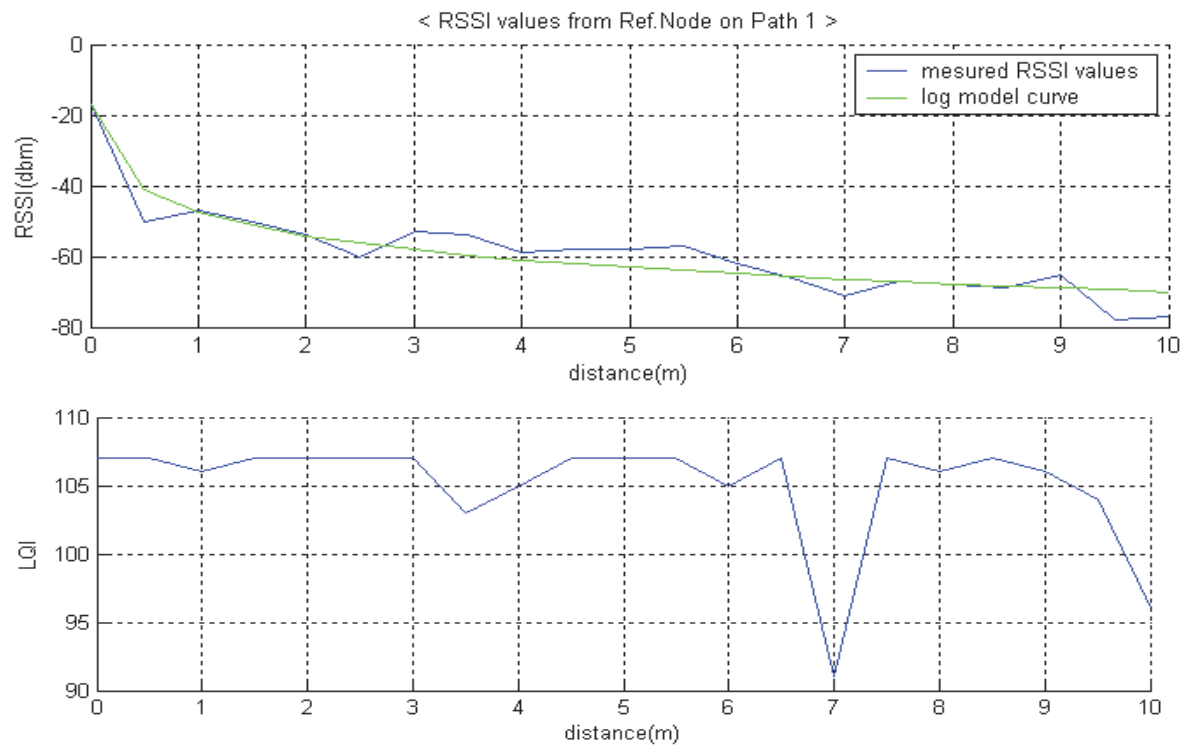

Fig. 7. Characteristics of RSSI and LQI in close space indoor environment. 
The proposed system was implemented with TinyOS 2.x and the ZigBee device (Hybus, Hmote 2430), which were designed to operate in environments where they are approximately co-planar, therefore constrained in 3 of their 6 degrees of freedom (pitch, roll and z-axis). Hardware had already been developed as a research platform. This section will describe these devices. The performance of the systems has been analyzed by implementation and simulation using Matlab.

\section{LQI and BOTH Filter}

The proposed scheme performs with respect to the signal analysis to filter the original signals in order to remove the noise. Sudden peaks and gaps in the signal strength are removed and the whole signal is smoothed, which eases the analysis process.

Various filters can be used to smooth the RSSI value. Two common filters are, simple averaging and feedback filters. Averaging is the most basic filter type, but it requires more data packets to be sent. Feedback filters uses only a small part of the most recent RSSI value for each calculation. This requires less data, but increases the latency when calculating a new position.

Averaging filter: The average RSSI value is simply calculated by requiring a few packets from each reference node, each time the RSSI value are measured and calculated according to the equation below:

$$
\overline{R S S I}=\frac{1}{n} \sum_{i=0}^{i=n} R S S I_{i}
$$

Feedback filter: If a filter approximation is used, this can be done as shown below. In this equation the variable $a$ is typically 0.75 or above. This approach ensures that a large difference in RSSI values will be smoothed. Therefore, it is not advisable if the assets that should be tracked can move long distance between each calculation.

$$
\operatorname{RSSI}_{n}=a * \operatorname{RSSI}_{n}+(1-a) * \operatorname{RSSI}_{n-1}
$$

This means that the averaged RSSI value corresponding to the signal strength at distance depends on both the previous averaged value and the most recently measured value. As the value of $a$, which should be between zero and one, determines the degree of filtering if $a$ is chosen to be close to one, the new measurement barely plays a role in the calculation of the new average. If on the other hand the value of $a$ is nearly zero, virtually no filtering is performed. An optimal filter, i.e., value for $a$, specific for this project will be determined in this section. In this section we are going to propose a new filtering process which is a fusion of RSSI and LQI where we decided to use LQI as a reference aid when the RSSI or LQI is below RSSI and LQI threshold, respectively.

Figures 8 and 9 represent the average distance error according to RSSI and LQI values for all three testbeds. Figure 8 shows that in 8 meters the LQI value is 100. From our experiment we found that when LQI $=100$, it gives $20 \%$ reliability. Whereas, Figure 9 shows that in 8 meters RSSI value is -77 , from our experiment we found that it gives $10 \%$ reliability. So we can come to a decision that over long distance LQI reliability is better than RSSI. Depend on this decision; we decide to use LQI as an assistance filtering factor for RSSI, which we are going to discuss next.

From Fig. 8, it is found that LQI gives best performance when the value was 108 in about 2 meters distance, which indicate that it gives $80 \%$ reliability. The results show that when the 
value is 100, it gives lowest performance in about 8 meters. As our measurement testbed was 10 meters, we decided to determine the value below 10 meters. So, we determined the LQI reliability from 100 to 108, where the reliability varies from $20 \%$ to $80 \%$, which means LQI filtering factor $a$ varies between 0.8 to 0.2 for LQI filtering. And we also determine that if $a$ value is below 101 then it should be negligible. The following equation has used for LQI filtering:

$$
\begin{gathered}
\text { smooth_RSSI } \mathrm{t}_{(\mathrm{LQI})}=\mathrm{a} * \operatorname{RSSI}_{\mathrm{t}}+(1-\mathrm{a}) * \mathrm{RSSI}_{\mathrm{t}-1} \\
a=0.8-0.6 * \frac{108-L Q I}{8}
\end{gathered}
$$

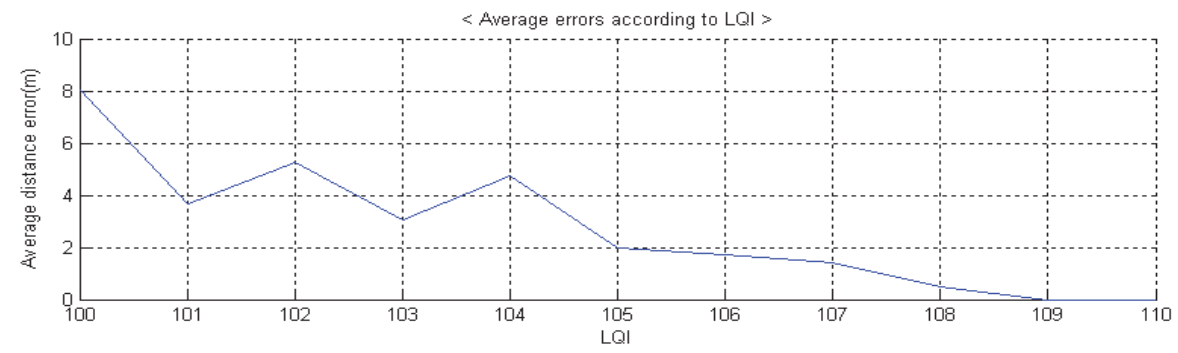

Fig. 8. Average errors according to LQI in all three paths.

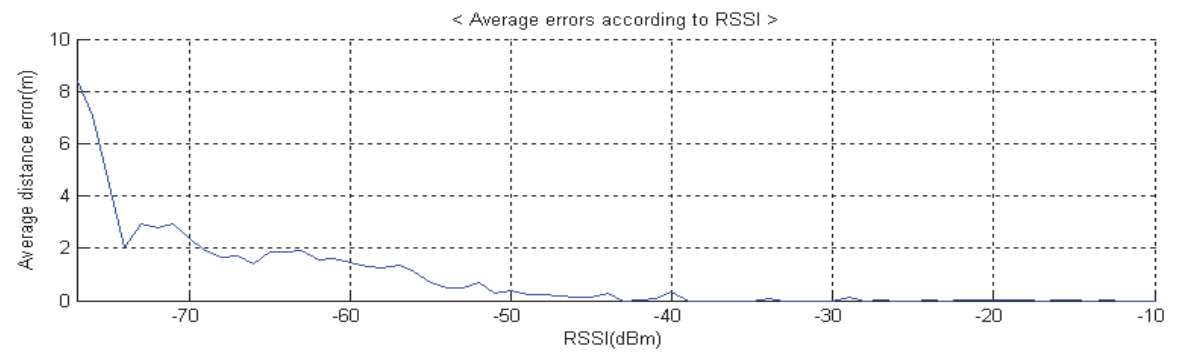

Fig. 9. Average errors according to RSSI in all three paths.

From Fig. 9, we found that RSSI gave best performance when its' value was -15 in about 0 meter distances, by which we determine that it gives $100 \%$ reliability. It has also seen that when its' value was -75 it gave lowest performance in about 5 meters, which means $50 \%$ reliability. So we decided the reliability for RSSI between -15 to -75 , where the reliability varies from $50 \%$ to $100 \%$. So this system decided to use the RSSI filtering factor $a$ around 1 to 0.5 according to RSSI value. We also determine that if RSSI value is below -75 then the RSSI reliability will be $10 \%$, which could be neglected. However, the following equation was used for RSSI filtering:

$$
\begin{gathered}
\text { smooth_RSSI }{ }_{t(\mathrm{RSSI})}=\mathrm{a}^{*} \operatorname{RSSI}_{\mathrm{t}}+(1-\mathrm{a}){ }^{*} \mathrm{RSSI}_{\mathrm{t}-1} \\
a=1-0.5 * \frac{-15-\mathrm{RSSI}}{60}
\end{gathered}
$$


With this experiment, we want to determine an optimal $a$ value for filtering the radio signal strengths. We want to have a filter that is able to remove the noise i.e., the sudden peaks, gaps and shaded signal, but that should preserve the typical signal behavior of both stillness and movement.

For smoothing the real RSSI values, we use a fusion of RSSI and LQI filtering. For this BOTH filtering we use the LQI filtering in case of sudden peaks and shaded signals. In this BOTH filter we decided to measure the difference between present RSSI value and previous RSSI value. If the difference of present and previous RSSI value smaller than RSSI threshold value or if the LQI value is smaller than LQI threshold value then the signal will be filtered by RSSI filter otherwise it will use LQI filter. To determine the LQI threshold value, we use the defined LQI threshold value 105. For determining RSSI threshold value, we use ADD (average distance difference) value as a RSSI threshold average distance difference (ADD) [27].

At first we used LQI filtering technique to smooth the absolute RSSI values and after that we used BOTH filtering process.

\section{LQI Filter}

For smoothing the real RSSI values we use LQI filtering, so the real RSSI has smoothed very well.

$$
\begin{gathered}
\text { if } L Q I<100 \\
a=0 \\
\text { else } \\
\text { smooth_RSSI } I_{t(L Q I)}=a * \operatorname{RSSI}_{t}+(1-a) * R^{2} S_{t-1}
\end{gathered}
$$

After applying the filter, the program analyses the behavior of the filtered signal strengths over distance. Following figures provide the results:

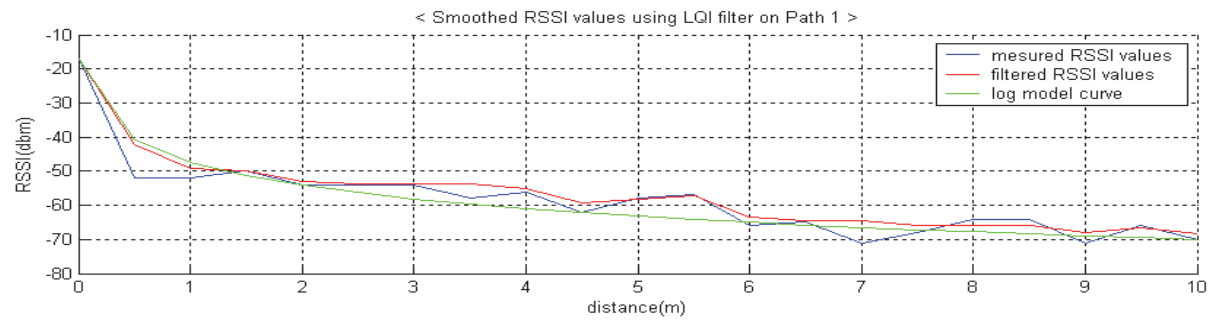

Fig. 10. Smoothed RSSI values using LQI filter for path 1.

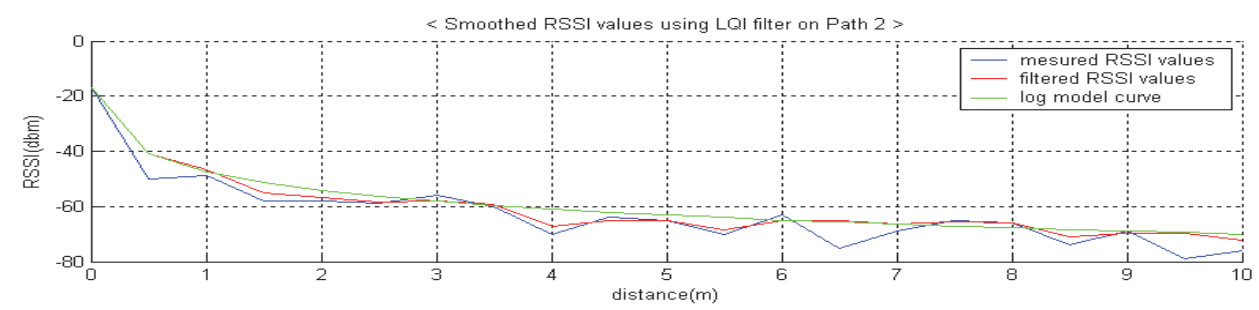

Fig. 11. Smoothed RSSI values using LQI filter for path 2. 


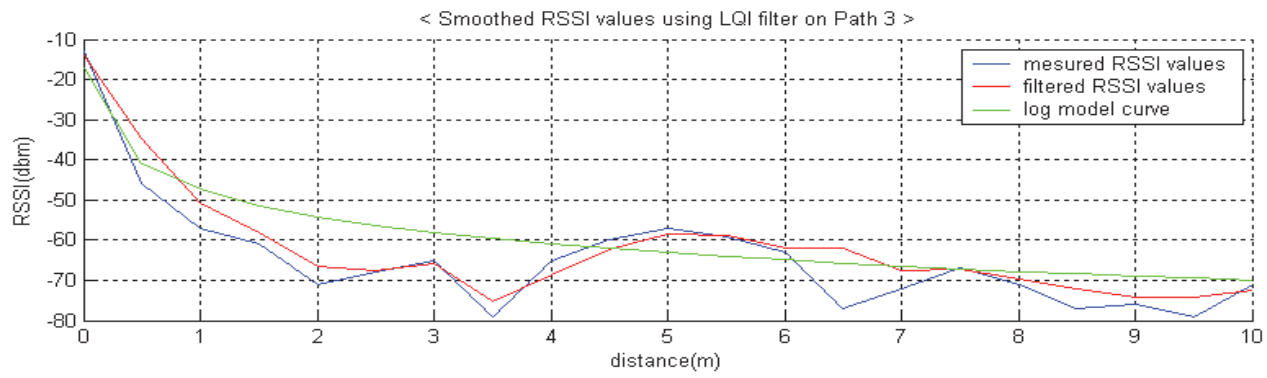

Fig. 12. Smoothed RSSI values using LQI filter for path 3.

\section{BOTH Filter}

The following equation has used to filter both RSSI and LQI values:

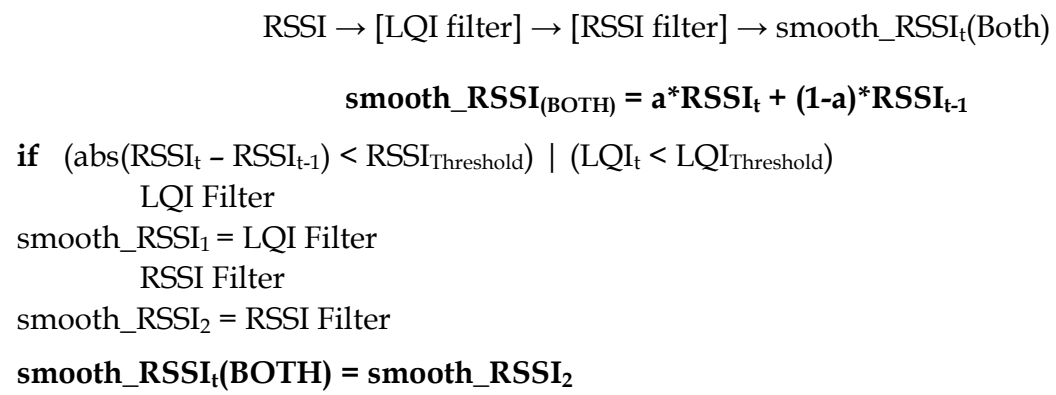

After applying the filter, the program analyses the behavior of the filtered signal strengths over distance. Based on analysis, this system decides LQI values perform better than RSSI values over distance in a fully indoor environment. But on the other two environments, RSSI values perform better than LQI values. The best performance is happened when the RSSI values smoothed by BOTH filtering. Following figures provide the results:

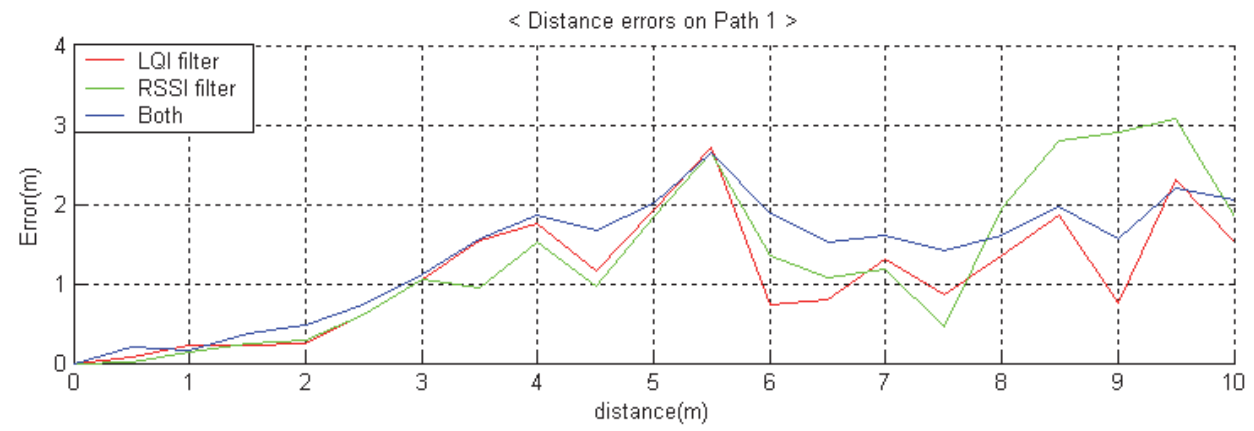

Fig. 13. Smoothed RSSI values using LQI, RSSI and BOTH filter for path 1. 
From the above analyses, we can determine that BOTH filters perform better than the existing RSSI filter [4], Fusion filter [27] and our proposed LQI filter.

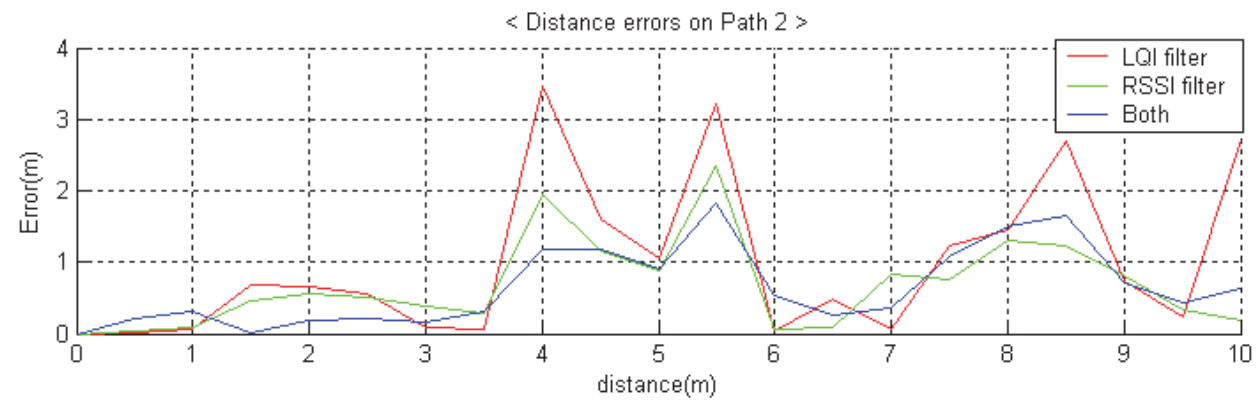

Fig. 14. Smoothed RSSI values using LQI, RSSI and BOTH filter for path 2.

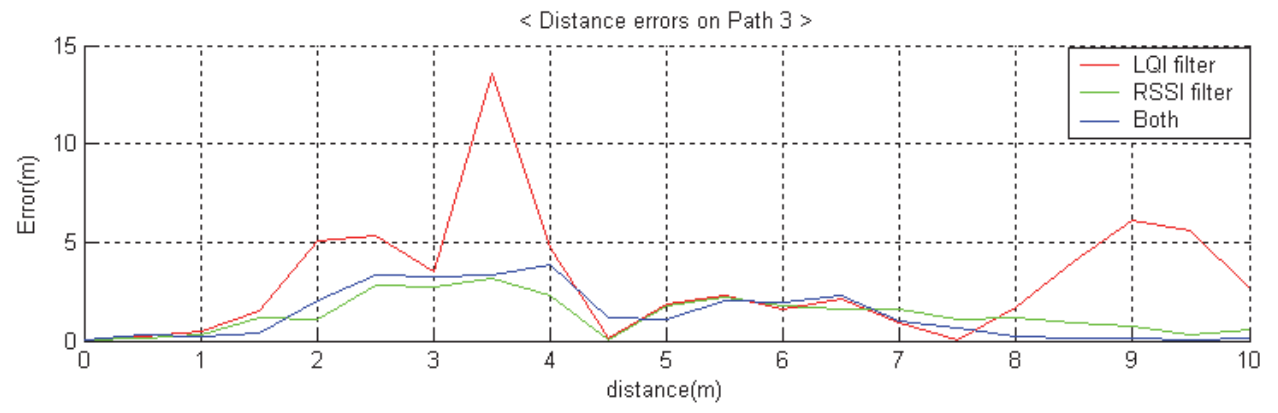

Fig. 15. Smoothed RSSI values using LQI, RSSI and BOTH filter for path 3.

\section{Experimental performance}

From the above findings, we can say that the proposed BOTH filtering algorithm could reduce more average error and maximum error distance than other existing algorithm [4], [12], [27], [28]. The average reduction of distance error deduced by using the proposed BOTH filtering is $56 \%$ and $68 \%$ average error and maximum error, respectively than the existing RSSI filtering [4] and Fusion filtering[27]. So we can say that our proposed method can perform well over other existing algorithm.

Table 3 shows the experimental results of our proposed filtering algorithm. From adaptive adjustment we found that this process could reduce a high number of errors from without filter measured signal.

From above discussion, we can draw the conclusion that our new enhancement technique gives a significantly improved performance over other existing techniques.

\section{Conclusion}

The technology is developing faster and faster, the expanding of the Internet makes people connected. There have been many forms of connections in cyberspace i.e., wired connection, wireless network, structured network, Ad-hoc network, etc. Life will be rather different without any form of such communication. Certainly the security will be a great concern in 


\begin{tabular}{|c|c|c|c|c|c|c|c|c|c|c|c|}
\hline 焉 & $\begin{array}{l}\text { FILTER } \\
\text { NAME }\end{array}$ & $\begin{array}{c}\text { AVG. } \\
\text { ERROR }\end{array}$ & $\begin{array}{l}\text { MAX } \\
\text { ERROR }\end{array}$ & \multicolumn{2}{|c|}{$\begin{array}{c}\text { RSSI } \\
\text { FILTER }\end{array}$} & \multicolumn{2}{|c|}{$\begin{array}{c}\text { LQI } \\
\text { FILTER }\end{array}$} & \multicolumn{2}{|c|}{$\begin{array}{c}\text { FUSION } \\
\text { FILTER }\end{array}$} & \multicolumn{2}{|c|}{ BOTH FILTER } \\
\hline \multirow{5}{*}{1} & Non filter & 1.2750 & 4.0577 & \multirow{6}{*}{$\begin{array}{c}\text { Avg. } \\
\text { RDC. } \\
\text { of } \\
\text { Avg. } \\
\text { Error }\end{array}$} & \multirow{6}{*}{$\begin{array}{l}\text { Avg. } \\
\text { RDC. } \\
\text { of } \\
\text { Avg. } \\
\text { Error }\end{array}$} & \multirow{6}{*}{$\begin{array}{l}\text { Avg. } \\
\text { RDC. } \\
\text { of } \\
\text { Avg. } \\
\text { Error }\end{array}$} & \multirow{6}{*}{$\begin{array}{l}\text { Avg. } \\
\text { RDC. } \\
\text { of } \\
\text { Avg. } \\
\text { Error }\end{array}$} & \multirow{6}{*}{$\begin{array}{c}\text { Avg. } \\
\text { RDC. } \\
\text { of } \\
\text { Avg. } \\
\text { Error }\end{array}$} & \multirow{6}{*}{$\begin{array}{c}\text { Avg. } \\
\text { RDC. } \\
\text { of } \\
\text { Avg. } \\
\text { Error }\end{array}$} & \multirow{6}{*}{$\begin{array}{l}\text { Avg. } \\
\text { RDC. } \\
\text { of } \\
\text { Avg. } \\
\text { Error }\end{array}$} & \multirow{6}{*}{$\begin{array}{l}\text { Avg. } \\
\text { RDC. } \\
\text { of } \\
\text { Avg. } \\
\text { Error }\end{array}$} \\
\hline & $\begin{array}{l}\text { RSSI } \\
\text { Filter }\end{array}$ & 1.0911 & 3.1117 & & & & & & & & \\
\hline & $\begin{array}{c}\text { LQI } \\
\text { Filter } \\
\end{array}$ & 1.0962 & 2.7127 & & & & & & & & \\
\hline & $\begin{array}{l}\text { Fusion } \\
\text { Filter }\end{array}$ & 1.2807 & 3.0742 & & & & & & & & \\
\hline & $\begin{array}{l}\text { BOTH } \\
\text { filter }\end{array}$ & 1.0959 & 2.6453 & & & & & & & & \\
\hline \multirow{5}{*}{2} & Non filter & 3.0142 & 15.183 & & & & & & & & \\
\hline & $\begin{array}{l}\text { RSSI } \\
\text { Filter }\end{array}$ & 1.0943 & 5.4847 & \multirow{9}{*}{$33 \%$} & \multirow{9}{*}{$50 \%$} & \multirow{9}{*}{$41 \%$} & \multirow{9}{*}{$49 \%$} & \multirow{9}{*}{$49 \%$} & \multirow{9}{*}{$62 \%$} & \multirow{9}{*}{$56 \%$} & \multirow{9}{*}{$68 \%$} \\
\hline & $\begin{array}{c}\text { LQI } \\
\text { Filter } \\
\end{array}$ & 1.0040 & 3.4574 & & & & & & & & \\
\hline & $\begin{array}{c}\text { Fusion } \\
\text { Filter }\end{array}$ & 0.8408 & 3.3281 & & & & & & & & \\
\hline & $\begin{array}{l}\text { BOTH } \\
\text { filter }\end{array}$ & 0.6495 & 1.8214 & & & & & & & & \\
\hline \multirow{5}{*}{3} & Non filter & 5.3016 & 21.183 & & & & & & & & \\
\hline & $\begin{array}{l}\text { RSSI } \\
\text { Filter }\end{array}$ & 2.3972 & 7.2861 & & & & & & & & \\
\hline & $\begin{array}{c}\text { LQI } \\
\text { Filter } \\
\end{array}$ & 3.0213 & 13.609 & & & & & & & & \\
\hline & $\begin{array}{c}\text { Fusion } \\
\text { Filter }\end{array}$ & 1.3088 & 3.1259 & & & & & & & & \\
\hline & $\begin{array}{l}\text { BOTH } \\
\text { filter }\end{array}$ & 1.3174 & 3.8351 & & & & & & & & \\
\hline
\end{tabular}

Table 3. Error reduction comparison of RSSI filter, Fusion filter, proposed LQI filter and BOTH filter

such beneficial technology. The security measures to provide Confidentiality and Integrity have been taken into account in the design of such technology. This chapter investigates the use of RF location systems for indoor domestic applications. Based on the assumption, low cost and minimal infrastructure are important for consumers, the concept of RF location system for Integrated In-door Location Using RSSI and LQI provided by ZigBee module is introduced.

This chapter addresses the problem of tracking an object. This chapter discuss about how to overcome the problems in the existing methods calculating the distance in indoor environment. This chapter has presented a new Mathematical Method for reducing the error in the location identification due to interference within the infrastructure based sensor 
network. The proposed Mathematical Method calculates the distance using LQI and RSSI predicted based on the previously measured values. The calculated distance corrects the error induced by interference. The experimental results show that the proposed Mathematical Method can reduce the average error around 25\%, and it is always better than the other existing interference avoidance algorithms. This technique has been found to work well in instances modeled on real world usage and thereby minimizing the effect of the error and hope that the findings in this chapter will be helpful for design and implementation of object tracking system in indoor environment.

\section{Acknowledgements}

This work is financially supported by Korea Minister of Ministry of Land, Transport and Maritime Affairs (MLTM) as U-City Master's and Doctoral Course Grant Program. And special thanks to Yen Sethia for her kind cooperation.

\section{References}

[1] IEEE Standard for Information Technology. (October 2003). Wireless Medium Access Control (MAC) and Physical Layer (PHY) Specifications for Low-Rate Wireless Personal Area Networks (LR-WPANs), Local and Metropolitan Area Networks, Part 15.4

[2] Kamran, J. (January 2005). ZigBee Suitability for Wireless Sensor Networks in Logistic Telemetry Applications. Master's Thesis in Electrical Engineering, School of Information Science, Computer and Electrical Engineering, Halmstad University, Sweden

[3] Liu, H.; Darabi, H.; Banarjee, P. \& Liu, J. (2007). Survey of Wireless Indoor Positioning Techniques and Systems. IEEE Transactions on Systems, Man, and Cybernetics-Part C: Applications and Reviews, Vol.37, No.6, (2007), pp. 1067-1080

[4] Tae Young, C. (December 2007). A Study on In-door Positioning Method Using RSSI Value in IEEE 802.15.4 WPAN. Master's Thesis in School of Electronical Engineering \& Computer Science, Kyungpook National University, Korea

[5] http://www.ZigBee.org/en/about/faq.asp

[6] Dragos, N. \& Badri, N. (April 2001). Ad-hoc Positioning System, Technical Report DCSTR-435, Rutgers University, also in Symposium on Ad-Hoc Wireless Networks, pp. 2926-2931, San Antonio, Texas, USA, November 2001

[7] Lorincz, K. \& Welsh, M. (2005). Motetrack: A Robust, Decentralized Aproachto RF-based Location Tracking, Proceedings of the International Workshop on Location- and ContextAwareness (LoCA '05), Munich, Germany, May 12-13, 2005

[8] Vehbi Cagri, G. (August 2007). Real-Time and Reliable Communication Inwireless Sensor and Actor Networks. PhD Thesis in School of Electrical and Computer Engineering, Georgia Institute of Technology, USA

[9] Zhang, J.; Yan, T.; Stankovic, J. \& Son, S. (2005). Thunder: A Practical Acoustic Localization Scheme for Outdoor Wireless Sensor Networks. Technical Report CS2005-13, Department of Computer Science, University of Virginia, USA

[10] Priyantha, N.; Chakraborty, A. \& Balakrishnan, H. (2000). The Cricket Location-Support System, Proceedings of the $6^{\text {th }}$ Annual International Conference on Mobile Computing and Networking, pp. 32-43, Boston, MA, USA, August 6-11, 2000 
[11] Alippi, C. \& Vanini, G. (2005). A RF Map-based Localization Algorithm for Indoor Environments, Proceedings of the IEEE International Symposium on Circuits and Systems, pp. 652-655, Kobe, Japan, May 23-26, 2005

[12] Bahl, P. \& Padmanabhan, V. RADAR: An In-building RF-based User Location and Tracking System. INFOCOM, Vol.2, pp. 775-784, Tel Aviv, Israel

[13] Kumar, S. (February 2006). Sensor System for Positioning and Identification in Ubiquitous Computing. Final Thesis

[14] Bulusu, N.; Heidemann, J. \& Estrin, D. (2000). GPS-less Low Cost Outdoor Localization for Very Small Devices, Personal Communications Magazine, Vol.7, No.5, pp. 2834, Octobar 2000

[15] Kaemarungsi, K. (2005). Design of Indoor Positioning System Based on Location Fingerprint Technique. Master's thesis, University of Pittsburgh, USA

[16] http:/ / www.uk.research.att.com/bat/

[17] CC2430 datasheet. Available from http://www.chipcon.com/

[18] Want, R.; Hopper, A.; Falcao, V. \& Gibbons, J. The Active Badge Location System. Technical Report 92.1, Olivetti Research Limited (ORL), ORL, 24a Trumpington Street, Cambridge CB2 1QA, UK

[19] Krohn, A.; Beigl, M.; Hazas, M.; Gellersen, H. \& Schmidt, A. (2005). Using Fine-grained Infrared Positioning to Support the Surface Based Activities of Mobile Users, Fifth International Workshop on Smart Appliances and Wearable Computing (IWSAWC), Columbus, Ohio, USA, June 10, 2005

[20] http://www.ubisense.net/

[21] Fukuju, Y.; Minami, M.; Morikawa, H. \& Aoyama, T. (2003). Dolphin: An Autonomous Indoor Positioning System in Ubiquitous Computing Environment, IEEE Workshop on Software Technologies for Future Embedded Systems (WSTFES2003), pp. 53-56, Hakodate, Hokkaido, Japan, May 2003

[22] Priyantha, N.; Miu, A.; Balakrishnan, H. \& Teller, S. (2001). The Cricket Compass for Context-aware Mobile Applications, Proceedings of the $7^{\text {th }}$ Annual International Conference on Mobile Computing and Networking, pp. 1-14, Rome, Italy, July 16-21, 2001

[23] Bahl, P.; Padmanabhan, V. \& Balacgandran, A. (2000). Enhancements to the RADAR User Location and Tracking System. Microsoft Research Technical Report, February 2000.

[24] Getting, I. The Global Positioning System. IEEE Spectrum, Vol.30, No.12, (December 1993), pp. 36- 47,

[25] Simon, H. (1984). Introduction to Adaptive Filters, ISBN 0029494605, Collier Macmillan Publishers, London

[26] Sayed, A. (2003). Fundamentals of Adaptive Filtering, ISBN 0471461261, IEEE Press WileyInterscience, New York

[27] Halder, S. J.; Choi, T.; Park, J.; Kang, S.; Park, S. \& Park, J. (2008). Enhanced Ranging Using Adaptive Filter of ZIGBEE RSSI and LQI Measurement, Proceedings of The 10th International Conference on Information Integration and Web-based Applications $\mathcal{E}$ Services (iiWAS2008), pp. 367-373, Linz, Austria, November 24-26, 2008

[28] Halder, S. J.; Choi, T.; Park, J.; Kang, S.; Yun, S. \& Park, J. (2008). On-line Ranging for Mobile Objects Using ZIGBEE RSSI Measurement. Proceedings of The 3rd International Conference on Pervasive Computing and Applications (ICPCA2008), pp. 662-666, Alexandria, Egypt, October 06-08, 2008 


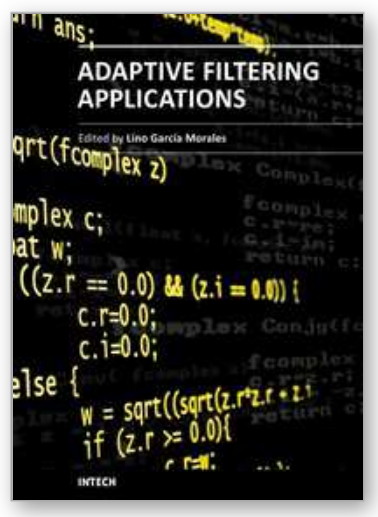

\author{
Adaptive Filtering Applications \\ Edited by Dr Lino Garcia
}

ISBN 978-953-307-306-4

Hard cover, 400 pages

Publisher InTech

Published online 24, June, 2011

Published in print edition June, 2011

Adaptive filtering is useful in any application where the signals or the modeled system vary over time. The configuration of the system and, in particular, the position where the adaptive processor is placed generate different areas or application fields such as: prediction, system identification and modeling, equalization, cancellation of interference, etc. which are very important in many disciplines such as control systems, communications, signal processing, acoustics, voice, sound and image, etc. The book consists of noise and echo cancellation, medical applications, communications systems and others hardly joined by their heterogeneity. Each application is a case study with rigor that shows weakness/strength of the method used, assesses its suitability and suggests new forms and areas of use. The problems are becoming increasingly complex and applications must be adapted to solve them. The adaptive filters have proven to be useful in these environments of multiple input/output, variant-time behaviors, and long and complex transfer functions effectively, but fundamentally they still have to evolve. This book is a demonstration of this and a small illustration of everything that is to come.

\title{
How to reference
}

In order to correctly reference this scholarly work, feel free to copy and paste the following:

Sharly Joana Halder, Joon-Goo Park and Wooju Kim (2011). Adaptive Filtering for Indoor Localization using ZIGBEE RSSI and LQI Measurement, Adaptive Filtering Applications, Dr Lino Garcia (Ed.), ISBN: 978-953307-306-4, InTech, Available from: http://www.intechopen.com/books/adaptive-filtering-applications/adaptivefiltering-for-indoor-localization-using-zigbee-rssi-and-lqi-measurement

\section{INTECH}

open science | open minds

\section{InTech Europe}

University Campus STeP Ri

Slavka Krautzeka 83/A

51000 Rijeka, Croatia

Phone: +385 (51) 770447

Fax: +385 (51) 686166

www.intechopen.com

\section{InTech China}

Unit 405, Office Block, Hotel Equatorial Shanghai

No.65, Yan An Road (West), Shanghai, 200040, China

中国上海市延安西路65号上海国际贵都大饭店办公楼405单元

Phone: +86-21-62489820

Fax: +86-21-62489821 
(C) 2011 The Author(s). Licensee IntechOpen. This chapter is distributed under the terms of the Creative Commons Attribution-NonCommercialShareAlike-3.0 License, which permits use, distribution and reproduction for non-commercial purposes, provided the original is properly cited and derivative works building on this content are distributed under the same license. 